\title{
Tenofovir-based alternate therapies for chronic hepatitis B patients with partial virological response to entecavir
}

\author{
L. Lu, ${ }^{1,2}$ B. Yip, ${ }^{3}$ H. Trinh, ${ }^{4}$ C. Q. Pan, ${ }^{5}$ S.-H. B. Han, ${ }^{6}$ C. C. Wong, ${ }^{7}$ J. Li, ${ }^{8}$ S. Chan, ${ }^{9}$ \\ G. Krishnan, ${ }^{10}$ C. C. Wong ${ }^{11}$ and M. H. Nguyen ${ }^{2}{ }^{1}$ Medical School, University of Michigan Medical School, Ann \\ Arbor, MI, USA; ${ }^{2}$ Division of Gastroenterology and Hepatology, Stanford University Medical Center, Palo Alto, CA, USA; ${ }^{3}$ Medical School, Eastern \\ Virginia Medical School, Norfolk, VA, USA; ${ }^{4}$ San Jose Gastroenterology, San Jose, CA, USA; ${ }^{5}$ Division of Gastroenterology and Hepatology, NYU \\ Langone Medical Center, NYU School of Medicine, New York, NY, USA; ${ }^{6}$ Pfleger Liver Institute, Ronald Reagan UCLA Medical Center, Los Angeles, \\ CA, USA; ${ }^{7}$ Christopher Wong, MD, San Francisco, CA, USA; ${ }^{8}$ Palo Alto Medical Foundation, Mountain View, CA, USA; ${ }^{9}$ Medical Procare, \\ Flushing, NY, USA; ${ }^{10}$ Stanford Center for Clinical Informatics, Stanford University School of Medicine, Stanford, CA, USA; and ${ }^{11}$ Clifford Wong, \\ MD, San Francisco, CA, USA
}

Received August 2014; accepted for publication October 2014

SUMMARY. Entecavir (ETV) is a first-line antiviral therapy for treating chronic hepatitis $\mathrm{B}(\mathrm{CHB})$; however, some patients have suboptimal response to ETV. Currently, there are limited data on how to approach these patients. Therefore, our aim was to compare the effectiveness of two alternate therapies - tenofovir (TDF) monotherapy and combination therapy of ETV+TDF - in CHB patients with ETV partial virological response. We conducted a retrospective study of 68 patients who had partial virological response to ETV, defined as having detectable HBV DNA following at least 12 months of ETV, and were switched to TDF monotherapy $(n=25)$ or ETV+TDF $(n=43)$. Patients were seen in seven US liver/community-based clinics and started on ETV between 2005 and 2009. The majority of patients were male; the vast majority were Asian and had positive hepatitis B e antigen ( $\mathrm{HBeAg}$ ). Patients in both groups had similar pretreatment characteristics. Complete viral suppression (CVS) rates with TDF monotherapy and ETV+TDF were similar after 6 months $(71 \%$ vs $83 \%$, $P=0.23)$ and 12 months $(86 \%$ vs $84 \%, P=0.85)$, and there was no statistically significant difference in CVS rates even when only patients with higher HBV DNA levels at switch $(>1000 \mathrm{IU} / \mathrm{mL})$ were evaluated. Multivariate analysis indicated that ETV+TDF was not an independent predictor of CVS compared to TDF monotherapy ( $\mathrm{OR}=1.19$, $P=0.63)$. In conclusion, TDF monotherapy and ETV + TDF are comparable in achieving CVS in CHB patients with partial virological response to ETV. Long-term alternate therapy with one pill (TDF monotherapy) vs two pills $(\mathrm{ETV}+\mathrm{TDF})$ could lead to lower nonadherence rates and better treatment outcomes.

Keywords: chronic hepatitis B, entecavir, partial virological response, switch, tenofovir.

\section{INTRODUCTION}

According to the WHO, chronic hepatitis B (CHB) affects more than 240 million people worldwide, and hepatitis B virus (HBV) infections lead to an estimated 600000

Abbreviations: ADV, adefovir; ALT, alanine aminotransferase; CHB, chronic hepatitis B; CVS, complete viral suppression; EASL, European Association for the Study of the Liver; ETV, entecavir; HBeAg, hepatitis B e antigen; HBV, hepatitis B virus; LAM, lamivudine; NYU, New York University; TDF, tenofovir; UCLA, University of California, Los Angeles.

Correspondence: Mindie H. Nguyen, MD, MAS, Associate Professor of Medicine, Division of Gastroenterology and Hepatology, Stanford University Medical Center, 750 Welch Road, Suite 210, Palo Alto, CA 94304, USA.

E-mail: mindiehn@stanford.edu deaths per year due to complications such as cirrhosis, hepatic decompensation and hepatocellular carcinoma [1-3]. Higher levels of viremia are associated with more rapid disease progression $[4,5]$; therefore, the primary goal of anti-HBV therapy is to achieve complete viral suppression (CVS) in order to prevent progression and ultimately premature death [6].

Currently, entecavir (ETV) and tenofovir (TDF) are the most effective anti-CHB agents based on their efficacy, tolerability and minimal rates of antiviral resistance $[7,8]$. Long-term studies show that ETV can successfully suppress $\mathrm{HBV}$ replication to undetectable HBV DNA levels in over $90 \%$ of hepatitis B e antigen (HBeAg)-negative patients $[8,9]$. However, in HBeAg-positive individuals with high levels of viremia, a significant portion of patients experience only partial virological response [3]. Studies of ETV monotherapy in HBeAg-positive patients have found that 
approximately $30 \%$ of patients were partial virological responders (detectable HBV DNA levels), 5\% were nonresponders (HBV DNA levels $\geq 10^{5}$ copies $/ \mathrm{mL}$ ), and $2 \%$ experienced a virological breakthrough after 48 weeks of ETV $[10,11]$. Treatment that fails to suppress viral replication can not only lead to progression of $\mathrm{CHB}$ but also to selection of antiviral resistance mutations [12].

Currently, data are limited regarding how to approach patients with partial virological responses to ETV. A recent study by Chen et al. [13] showed that the majority of patients with HBV DNA >2000 copies/mL after 48 weeks of ETV did not achieve CVS with additional ETV. Ha et al. [14] examined patients who remained viremic after at least 24 weeks of ETV at $0.5 \mathrm{mg}$ and observed that increasing to $1.0 \mathrm{mg}$ did not help the majority achieve CVS. On the other hand, a study by Pan et al. [15] of patients with partial virological response to ETV suggested that switching to TDF monotherapy could be an effective option. Another study by Petersen et al. [16] analysing patients who were partial virological responders reported that a combination of ETV+TDF was a safe and efficient treatment. One other study by Yip et al. [17] evaluating patients with positive viremia after at least 12 months of ETV concluded that patients did better when switched to TDF monotherapy or ETV+TDF compared to combination therapy of ETV and adefovir (ADV). However, the sample size was small in all of the above studies and thus limited their conclusions. Therefore, our goal was to assess the rates of CVS in a larger, multicentre cohort of $\mathrm{CHB}$ patients with partial virological response to ETV between two alternate therapies: TDF monotherapy and ETV+TDF combination therapy.

\section{MATERIALS AND METHODS}

\section{Study design and population}

We conducted a retrospective cohort study of 68 adult patients with $\mathrm{CHB}$ who were seen in liver clinics at Stanford University Medical Center, New York University (NYU) Langone Medical Center, Ronald Reagan University of California, Los Angeles (UCLA) Medical Center and four California community-based clinics in the San Francisco Bay Area. The study protocol was approved by the Institution Review Boards at Stanford, NYU, and UCLA. Eligible patients were CHB patients who had partial virological response to ETV, defined as having detectable HBV DNA levels $(>60 \mathrm{IU} / \mathrm{mL})$ at the time of treatment modification following at least 12 months of ETV monotherapy, and were switched by their providing physician to either TDF monotherapy or combination therapy of ETV and TDF.

Patients were identified through an electronic query using the ICD-9 diagnosis code for $\mathrm{CHB}$ or by manual review of clinic patient $\operatorname{logs}$ and verified through chart review or case-based abstraction. Patients who demonstrated treatment nonadherence or developed viral resistance to ETV were excluded. ETV resistance was ruled out in 61 patients by negative genotypic mutation analysis and in seven patients by the absence of a virological breakthrough (increase $>1 \log _{10} \mathrm{IU} / \mathrm{mL}$ from nadir) while on ETV. In addition, patients who had human immunodeficiency virus/hepatitis $\mathrm{C}$ virus co-infections, were on immunosuppressive therapy, had a history of solid organ transplant, or had systemic chemotherapy were excluded.

\section{Statistical analysis}

Categorical variables were reported as proportions (\%), and continuous variables as mean $( \pm \mathrm{SD})$ or median (range). Categorical variables were evaluated using the chi-square test, while continuous variables were evaluated using the Student's $t$-test if a normal distribution was observed. If a normal distribution was not observed, then nonparametric methods were used for evaluation. The Kaplan-Meier method was used to estimate cumulative rates of CVS, and comparative analysis was performed using the log-rank test. Multivariate logistic regression was used to estimate odds ratios relating various baseline characteristics to the outcome of CVS rates between the two alternate therapy groups. Statistical significance was defined as a two-tailed $P$-value of 0.05 or less. All statistical analysis was performed using Stata 11.2 (StataCorp, College Station, TX, USA).

\section{RESULTS}

\section{Patient characteristics}

Of the two alternate therapy groups, 25 patients were switched to TDF monotherapy and 43 patients were switched to ETV+TDF combination therapy. Patients in the two groups were similar with respect to median age (40 vs $40, P=0.42)$ and the proportion of male patients $(64 \%$ vs $63 \%, P=0.92$ ). Of note, the vast majority of patients in both groups were positive for $\mathrm{HBeAg}(88 \%$ vs $95 \%$, $P=0.26)$. Almost all were also Asian (96\%). In terms of prior treatment, more patients in the TDF group received other antiviral therapy prior to ETV, but the distribution was not statistically significant $(44 \%$ vs $23 \%, P=0.07)$. More patients in the TDF group were also exposed to lamivudine (LAM), but again the distribution was not statistically significant $(24 \%$ vs $12 \%, \quad P=0.18)$. Based on genotypic mutation analysis, a total of four patients in the TDF group and one in the ETV+TDF group had known LAM resistance.

Both alternate treatment groups were on ETV monotherapy for a significant duration of time (median of 21 months for TDF vs 25 months for combination) before being switched to the new regimens. Both TDF monotherapy and ETV+TDF groups had similar HBV DNA levels prior to any treatment $(7.74 \log 10 \mathrm{IU} / \mathrm{mL}$ vs $7.97 \log 10 \mathrm{IU} / \mathrm{mL}$ $P=0.43$ ) (Table 1); however, HBV DNA levels at the start 
of ETV monotherapy and at the start of alternate therapy were slightly lower in the TDF group vs the ETV+TDF group $(6.69 \log 10 \mathrm{IU} / \mathrm{mL}$ vs $7.71 \log 10 \mathrm{IU} / \mathrm{mL}, P=0.01$ and $3.10 \log 10 \mathrm{IU} / \mathrm{mL}$ vs $3.57 \log 10 \mathrm{IU} / \mathrm{mL}, P=0.05$, respectively). With regard to alanine aminotransferase (ALT) levels, both groups had similar median ALT levels prior to ETV monotherapy (47 vs $51 \mathrm{U} / \mathrm{L}, P=0.33$ ) and at the start of alternate therapy (29 vs $26 \mathrm{U} / \mathrm{L}, P=0.41)$. Lastly, both treatment groups were followed on alternate therapies for a significant length of time (median of 16 months and 17 months).

When patients were stratified based on length of ETV monotherapy prior to TDF or ETV+TDF therapies $(\leq 2$ years $v s>2$ years), the only characteristic in which there was a statistically significant difference was HBV DNA levels at the time of switch to an alternate therapy (3.61 $\log 10 \mathrm{IU} /$ $\mathrm{mL}$ vs $3.15 \log 10 \mathrm{IU} / \mathrm{mL}, P=0.04$ ) (Table 2).

\section{Treatment outcomes}

Cumulative rates of CVS in the two patient groups, TDF monotherapy and ETV+TDF combination therapy, are depicted in the Kaplan-Meier curves in Fig. 1. Log-rank analysis demonstrated no statistically significant difference between the two therapy groups. There was also no statistically significant difference in the rates of CVS of the two treatment groups in patients with HBV DNA levels $>1000 \mathrm{IU} / \mathrm{mL}$ at the time of switch (Fig. 2).

CVS rates after 6 months and 12 months of alternate therapy were also stratified by duration of prior ETV monotherapy: $\leq 2$ years and $>2$ years before switching to TDF or ETV+TDF therapy (Fig. 3). For patients on ETV for up to 2 years, CVS rates at 6 and 12 months were similar between the two therapies $(71 \%$ vs $70 \%, P=0.93$ and $77 \%$ vs $76 \%, P=0.98$, respectively). For patients on ETV monotherapy for longer than 2 years, CVS rates were lower for the TDF group after 6 months (70\% vs 95\%, $P=0.04$ ), but after 12 months, TDF monotherapy patients had higher CVS rates than the ETV+TDF combination therapy group ( $100 \%$ vs $90 \%, P=0.35)$.

When the CVS rates of TDF monotherapy and ETV+TDF combination therapy were compared without stratifying based on length of ETV monotherapy, there was no statistically significant difference in CVS rates over time (Fig. 4). After 6 months of new therapy, CVS rates were $71 \%$ for patients on TDF and $83 \%$ for patients on ETV+TDF $(P=0.23)$. After 12 months, CVS rates were nearly identical: $86 \%$ with TDF and $84 \%$ with ETV+TDF $(P=0.85)$.

Multivariate analysis inclusive of age, time on ETV, and HBV DNA levels (i) prior to any treatment, (ii) prior to ETV monotherapy and (iii) at switch to alternate therapy demonstrated that combination therapy of ETV+TDF was not an independent predictor of CVS compared to TDF monotherapy $(\mathrm{OR}=1.19, \mathrm{P}=0.63)$.

\section{DISCUSSION}

While ETV is one of the most effective antiviral drugs for treating CHB $[7,8]$, a small proportion of patients will have a partial virological response to ETV, especially those positive for HBeAg and high pretreatment HBV DNA levels. For these patients, it is unclear whether switching to TDF would suffice or an add-on approach with TDF is needed. In this current study, a cohort of 68 patients, who were mostly Asian (96\%) and HBeAg positive (93\%), with partial virological response to ETV after a median treatment duration of 24 months was switched to two alternate therapies: TDF monotherapy and combination therapy of ETV+TDF. CVS rates with TDF monotherapy and ETV+TDF were similar at both the 6-month (71\% vs $83 \%)$ and the

Table 1 Patient characteristics stratified by alternate therapy group [tenofovir (TDF) monotherapy or entecavir (ETV)+TDF combination therapy] in patients with a partial virological response to ETV

\begin{tabular}{llll}
\hline & TDF monotherapy $(n=25)$ & ETV and TDF $(n=43)$ & $P$-value \\
\hline Median age (years) & $40(19-75)$ & $40(26-69)$ & 0.42 \\
Male & $64 \%$ & $63 \%$ & 0.92 \\
HBeAg positive & $88 \%$ & $95 \%$ & 0.26 \\
Nä̈ve HBV DNA $\left(\log _{10} \mathrm{IU} / \mathrm{mL}\right)$ & $7.74 \pm 1.17$ & $7.97 \pm 0.90$ & 0.43 \\
Median time on ETV before switch (months) & $21(12-59)$ & $17(6-72)$ & 0.71 \\
Median time on alternate therapy (months) & $16(6-56)$ & $7.71 \pm 1.15$ & 0.53 \\
Pre-ETV monotherapy & & $51(17-156)$ & 0.01 \\
$\quad$ HBV DNA $\left(\log _{10} \mathrm{IU} / \mathrm{mL}\right)$ & $6.69 \pm 2.03$ & & 0.33 \\
$\quad$ Median ALT $(\mathrm{IU} / \mathrm{L})$ & $47(8-153)$ & $3.57 \pm 0.90$ & 0.05 \\
At switch & $3.10 \pm 0.95$ & $26(9-75)$ & 0.41 \\
$\quad$ HBV DNA $\left(\log _{10} \mathrm{IU} / \mathrm{mL}\right)$ & $29(12-61)$ & \\
$\quad$ Median ALT $(\mathrm{IU} / \mathrm{L})$ & & & \\
\hline
\end{tabular}

TDF, tenofovir; ETV, entecavir; HBeAg, hepatitis B e antigen; HBV, hepatitis B virus; ALT, alanine aminotransferase. 
Table 2 Patient characteristics stratified by duration of entecavir (ETV) monotherapy before switch to an alternate therapy [tenofovir (TDF) monotherapy or ETV+TDF combination therapy] in patients with a partial virological response to ETV

\begin{tabular}{llll}
\hline & ETV treatment $\leq 2$ years $(n=36)$ & ETV treatment $>2$ years $(n=32)$ & $P$-value \\
\hline Median age (years) & $40(19-68)$ & $40(20-75)$ & 0.21 \\
Male & $69 \%$ & $56 \%$ & 0.26 \\
HBeAg positive & $92 \%$ & $94 \%$ & 0.74 \\
Prior treatment & $28 \%$ & $34 \%$ & 0.56 \\
Naïve HBV DNA $\left(\log _{10} \mathrm{IU} / \mathrm{mL}\right)$ & $7.78 \pm 1.12$ & $8.00 \pm 0.86$ & 0.43 \\
Pre-ETV monotherapy & $7.35 \pm 1.28$ & $7.31 \pm 1.91$ & 0.91 \\
$\quad$ HBV DNA $\left(\log _{10} \mathrm{IU} / \mathrm{mL}\right)$ & $49(8-156)$ & $51(19-99)$ & 0.91 \\
$\quad$ Median ALT $(\mathrm{IU} / \mathrm{L})$ & $3.61 \pm 1.04$ & $3.15 \pm 0.76$ & 0.04 \\
At switch & $28(12-75)$ & $26(9-63)$ & 0.89 \\
$\quad$ HBV DNA $\left(\log _{10} \mathrm{IU} / \mathrm{mL}\right)$ & Median ALT $(\mathrm{IU} / \mathrm{L})$ & & \\
\hline
\end{tabular}

ETV, entecavir; TDF, tenofovir; HBeAg, hepatitis B e antigen; HBV, hepatitis B virus; ALT, alanine aminotransferase.

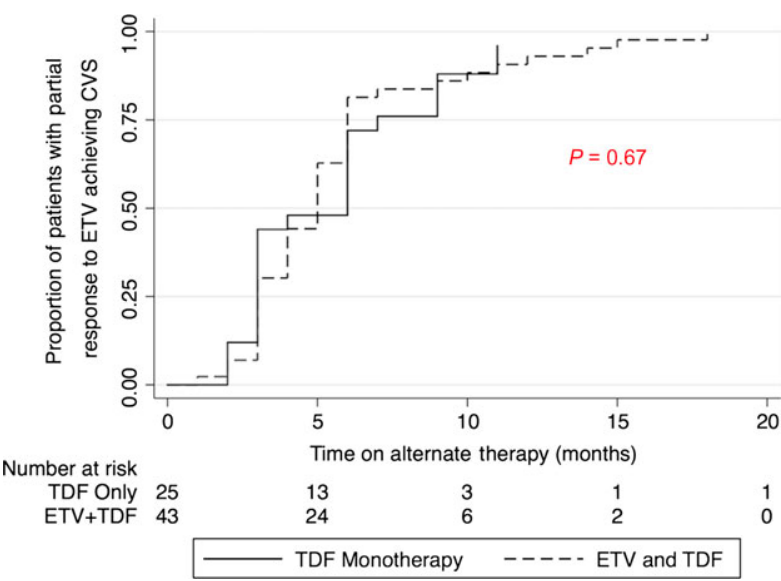

Fig. 1 Kaplan-Meier analysis of cumulative complete viral suppression (CVS) rates comparing tenofovir (TDF) monotherapy and entecavir (ETV) + TDF combination therapy as alternate therapies in patients with a partial virological response to ETV.

12-month ( $86 \%$ vs $84 \%)$ time points. In addition, there was no statistically significant difference in CVS rates between the two alternate therapies even when only patients with higher HBV DNA levels at the time of switch (>1000 IU/mL) were evaluated.

Current management guidelines by the European Association for the Study of the Liver (EASL) recommend that treatment adaptation be considered in patients with partial virological response to ETV at week 48, especially for those with nondeclining HBV DNA levels [18]. The median time on ETV in the current study was 21 months for the TDF group and 25 months for the ETV+TDF group, consistent with, and exceeding the EASL guidelines. One may argue that ETV monotherapy can be continued in this patient population, a treatment option assessed by Zoutendijk et al.

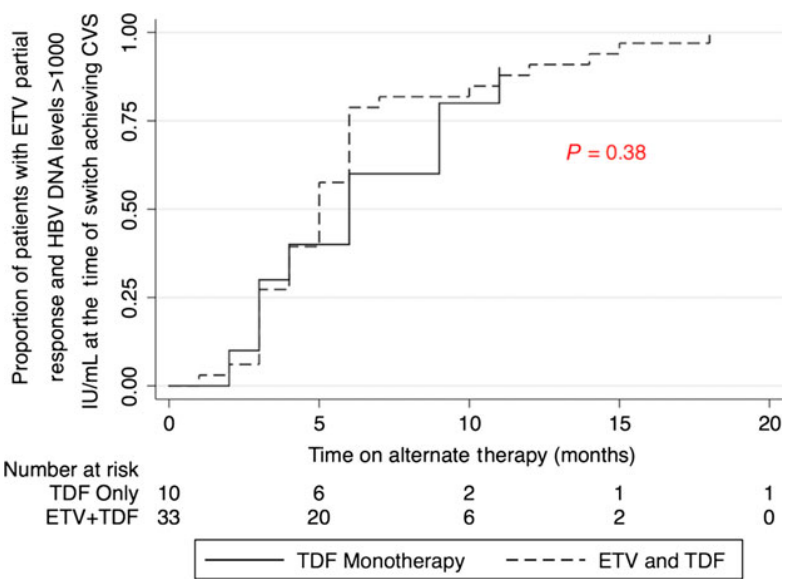

Fig. 2 Kaplan-Meier analysis of complete viral suppression (CVS) rates comparing tenofovir (TDF) monotherapy and entecavir (ETV) + TDF combination therapy as alternate therapies in patients with a partial virological response to ETV and HBV DNA levels $>1000 \mathrm{IU} / \mathrm{mL}$ at the time of switch.

[19]. In this study of European patients, the majority of ETV partial virological responders with low HBV viremia $(<1000 \mathrm{IU} / \mathrm{mL})$ after 48 weeks of ETV achieved CVS after 24 additional weeks of ETV. However, for those ETV partial virological responders with HBV DNA levels $>1000 \mathrm{IU} / \mathrm{mL}$ after 48 weeks of ETV monotherapy, $<10 \%$ achieved CVS after 48 additional weeks of ETV (totalling 2 years of ETV). In the current study, the majority of patients were treated with ETV monotherapy for a longer duration before being switched to an alternate therapy, and at the time of switch to an alternate therapy, the majority of these patients also had HBV DNA levels $>1000 \mathrm{IU} / \mathrm{mL}$.

A more recently published study by Chen et al. [13] analysed the effect of prolonged ETV monotherapy on 


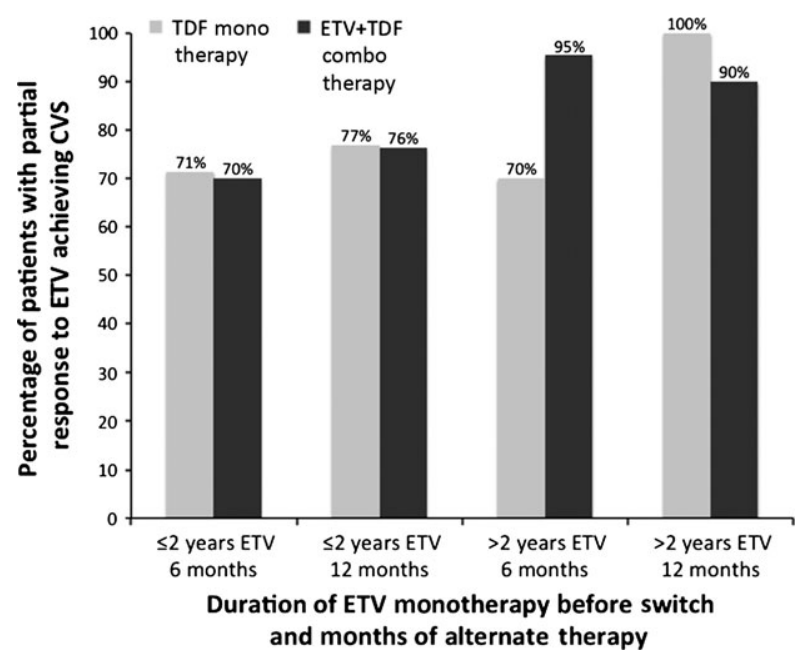

Fig. 3 Complete viral suppression (CVS) rates at 6 months and 12 months in patients with a partial virological response to entecavir (ETV) on alternate therapy of tenofovir (TDF) monotherapy or ETV+TDF combination therapy stratified by duration of ETV monotherapy: $\leq 2$ years $v s>2$ years.

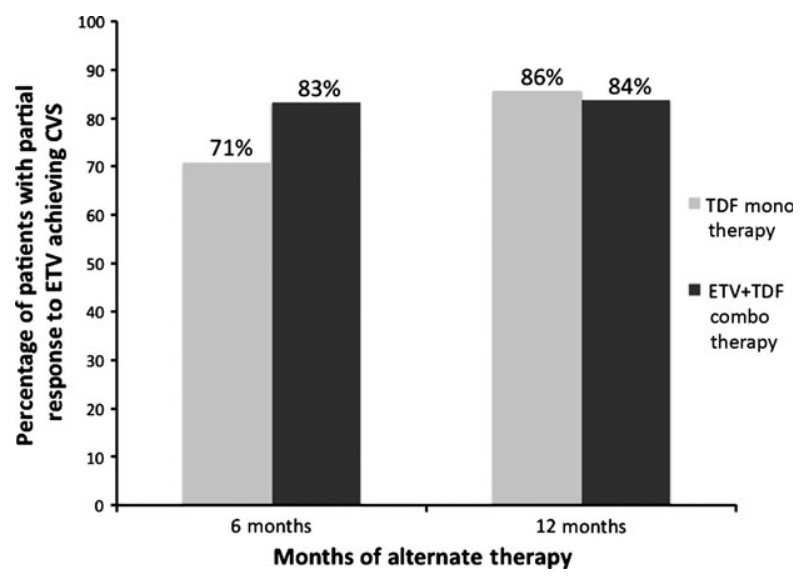

Fig. 4 Cumulative complete viral suppression (CVS) rates at 6 months and 12 months in patients with a partial virological response to entecavir (ETV) on alternate therapy of tenofovir (TDF) monotherapy or ETV+TDF combination therapy.

CHB patients with a partial virological response after 48 weeks of ETV. Among the subset of 369 patients who were treatment-naïve before ETV, 34 patients failed to achieve CVS after 48 weeks of ETV and were stratified based on HBV DNA levels greater or $<2000$ copies $/ \mathrm{mL}$ at 48 weeks. With continued ETV, $78 \%$ of patients with $<2000$ copies/mL achieved CVS with a median follow-up of 36 months (range 20 to 61 months), while only $27 \%$ of patients with $>2000$ copies $/ \mathrm{mL}$ achieved CVS with a median follow-up of 32 months (range 24 to
48 months). From these results, the authors concluded that prolonged ETV treatment for treatment-naïve patients with HBV DNA levels >2000 copies/mL after 48 weeks of ETV leads to a poor response and suggested that an alternate therapy, such as switching to TDF, be considered.

One other study assessing whether ETV monotherapy can be continued in patients who demonstrate partial virological responses to ETV was performed by Yang et al. [20]. In this study of 1254 Asian patients, while the majority achieved CVS, a significant minority (approximately 14\%) did not achieve CVS despite a median follow-up of 30 months (range 6-72 months). Among HBeAg-positive patients of this same study, the proportion of patients who did not achieve CVS over the duration of the study was higher at $20.6 \%$, further suggesting that some ETV-treated CHB patients may indeed require alternate therapies.

When considering alternate therapies, TDF monotherapy and combination therapy of ETV+TDF appeared comparable in terms of short-term effectiveness based on the results of the current study. However, TDF monotherapy may be the better long-term option due to potential issues of cost and nonadherence. Nonadherence, which prior studies have identified as one of the major causes of treatment failure [21,22], is of particular concern for $\mathrm{CHB}$, as antiviral treatment of this disease involves longterm commitment. In fact, for drugs with low viral resistance risk such as ETV and TDF, treatment failure may be attributed more commonly to nonadherence than to antiviral resistance. Therefore, a new alternate therapy with one pill (TDF monotherapy) vs two pills (ETV+TDF) could potentially lead to lower nonadherence rates and thus better overall treatment outcomes. While this study only measured outcomes after 6 and 12 months of alternate therapy, given the long-term treatment of CHB, potential benefits of TDF monotherapy over ETV+TDF combination therapy may be seen with longer follow-up.

Limitations to our study include its retrospective, nonrandomized design and a mostly Asian patient demographic.

In summary, for CHB patients who demonstrate partial virological responses to ETV, there was no statistically significant difference in CVS rates between TDF monotherapy and ETV+TDF combination therapy as a new alternate therapy. Given the economic advantage and the potentially higher adherence with monotherapy vs combination therapy, TDF monotherapy should be considered for ETV partial virological responders who desire alternate therapy. Although the current study is multicentre and larger than prior studies of ETV partial virological responders, the sample size of the study is still limited. Larger studies are needed to further evaluate the best long-term management strategy for this patient population. 


\section{CONFLICT OF INTERESTS}

Louis Lu has no financial relationship with a commercial interest. Benjamin Yip has no financial relationship with a commercial interest. Huy Trinh has served as a speaker for Vertex Pharmaceuticals and Bristol-Myers Squibb, consultant for Bristol-Myers Squibb, advisory board member for Gilead Sciences, Inc. and Bristol-Myers Squibb. He has received funding from Gilead Sciences, Inc. and Roche. He owns stock and shares in Gilead Sciences, Inc. Calvin Q. Pan has received research grants from Gilead Sciences, Inc. Bristol-Myers Squibb, Vertex Pharmaceuticals, Roche and Merck \& Co. He also serves as a consultant, advisor or speakers bureau for Gilead Sciences, Inc. Bristol-Myers Squibb, Vertex Pharmaceuticals, Onyx Pharmaceuticals and Janssen Pharmaceuticals Inc. Steven Han has served as a speaker, a consultant and an advisory board member for Gilead Sciences, Inc. and Bristol-Myers Squibb. He has also received research funding from Gilead Sciences, Inc. and Bristol-Myers Squibb. Christopher C. Wong has served as an advisory board member for Gilead Sciences, Inc. and Bristol-Myers Squibb. Jiayi Li has no financial relationship with a commercial interest. Stanley Chan has no financial relationship with a commercial interest. Clifford C. Wong has served as a speaker, a consultant and an advisory board member for Gilead Sciences, Inc. and Bristol-Myers Squibb. Gomathi Krishnan has no financial relationship with a commercial interest. Mindie H. Nguyen has served as a consultant for Bristol-Myers Squibb, Novartis Pharmaceuticals, Gilead Sciences, Inc., Bayer, and Onyx Pharmaceuticals and has received research funding from BristolMyers Squibb, Novartis Pharmaceuticals, Gilead Sciences, Inc. and Roche.

\section{FUNDING}

This study was funded in part by an NIH CTSA award number UL1 RR025744.

\section{REFERENCES}

1 Hepatitis B [Internet]. [Place unknown]:World Health Organization; [Date unknown] [updated 2013 July]. Available at: http://www.who. int/mediacentre/factsheets/fs204/en/ (accessed 6 June 2014).

2 Beasley RP. Hepatitis B virus. The major etiology of hepatocellular carcinoma. Cancer 1988; 61: 19421956.

3 Lok ASF, McMahon BJ. Chronic hepatitis B: update 2009. Hepatology 2009; 50: 661-662.

4 Iloeje UH, Yang HI, Su J et al. Predicting cirrhosis risk based on the level of circulating hepatitis B viral load. Gastroenterology 2006; 130: 678-686.

5 Liaw YF. Hepatitis B virus replication and liver disease progression: the impact of antiviral therapy. Antivir Ther 2006; 11: 669-679.

6 Mommeja-Marin H, Mondou E, Blum MR, Rousseau F. Serum HBV DNA as a marker of efficacy during therapy for chronic HBV infection: analysis and review of the literature. Hepatology 2003; 37: 13091319.

7 Woo G, Tomlinson G, Nishikawa Y et al. Tenofovir and entecavir are the most effective antiviral agents for chronic hepatitis B: a systematic review and bayesian meta-analyses.
Gastroenterology 2010; 139: $1218-$ 1229.

8 Keeffe EB, Dieterich DT, Han SH et al. A treatment algorithm for the management of chronic hepatitis B virus infection in the United States: 2008 update. Clin Gastroenterol Hepatol 2008; 6: 1315-1341.

9 Luo J, Li X, Wu Y et al. Efficacy of entecavir treatment for up to 5 years in nucleos( $\mathrm{t}$ )ide-Naïve chronic hepatitis B patients in real life. Int J Med Sci 2013; 10: 427433.

10 Gish R, Lok AS, Chang TT et al. Entecavir therapy for up to 96 weeks in patients with HBeAgpositive chronic hepatitis B. Gastroenterology 2007; 133: 1437-1444.

11 Colonno RJ, Rose R, Baldick CJ et al. Entecavir resistance is rare in nucleoside naïve patients with hepatitis B. Hepatology 2006; 44: 16561665.

12 Bartholomeusz A, Locarnini SA. Antiviral drug resistance: clinical consequences and molecular aspects. Semin Liver Dis 2006; 26: 162-170.

13 Chen $\mathrm{CH}$, Hu TH, Hung $\mathrm{CH}$, Wang JH, Lu SN, Lee CM. Antiviral effect of entecavir in nucleos(t)ide analogue-naïve and nucleos(t)ide analogue-experienced chronic hepatitis
B patients without virological response at week 24 or 48 of therapy. J Viral Hepat 2014; 21: e55e64.

14 Ha NB, Ha NB, Trinh HN, Nguyen $\mathrm{HA}$, Nguyen KK, Nguyen MH. Response to higher dose of entecavir $1.0 \mathrm{mg}$ daily in patients with partial response to entecavir $0.5 \mathrm{mg}$ daily. J Clin Gastroenterol 2013; 47: 461-465.

15 Pan CQ, Hu KQ, Yu AS, Chen W, Bunchorntavakul C, Reddy KR. Response to tenofovir monotherapy in chronic hepatitis B patients with prior suboptimal response to entecavir. J Viral Hepat 2012; 19: 213219.

16 Petersen J, Ratziu V, Buti M et al. Entecavir plus tenofovir combination as rescue therapy in pre-treated chronic hepatitis B patients: an international multicenter cohort study. $J$ Hepatol 2012; 56: 520-526.

17 Yip B, Chaung K, Wong CR et al. Tenofovir monotherapy and tenofovir plus entecavir combination as rescue therapy for entecavir partial responders. Dig Dis Sci 2012; 57: 3011-3016.

18 EASL clinical practice guidelines. Management of chronic hepatitis B virus infection. J Hepatol 2012; 57: 167-185. 
19 Zoutendijk R, Reijnders JG, Brown A et al. Entecavir treatment for chronic hepatitis B: adaptation is not needed for the majority of naïve patients with a partial virological response. Hepatology 2011; 54: 443-451.

20 Yang YJ, Shim JH, Kim KM, Lim YS, Lee HC. Assessment of current criteria for primary nonresponse in chronic hepatitis B patients receiving entecavir therapy. Hepatology 2014; 59: 1303-1310.

21 Ha NB, Ha NB, Garcia RT et al. Medication nonadherence with long-term management of patients with hepatitis B e antigen-negative chronic hepatitis B. Dig Dis Sci 2011; 56: 2423-2431.
22 Hongthanakorn C, Chotiyaputta W, Oberhelman $\mathrm{K}$ et al. Virological breakthrough and resistance in patients with chronic hepatitis B receiving nucleos(t)ide analogues in clinical practice. Hepatology 2011; 53: 1854-1863. 